\title{
Image-based determination of 'physiomarkers' for personalized cancer therapy
}
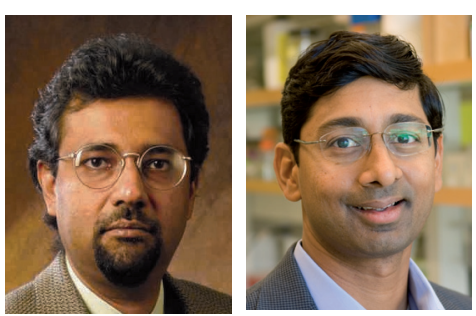

“...the physiomarker tumor vessel permeability is a significant determinant of drug dosing to tumors, and may have important clinical implications for a personalized diagnosis of the state of individual tumors."

\section{Ananth Annapragada \& Ravi V Bellamkonda ${ }^{\dagger}$}

${ }^{\dagger}$ Author for correspondence: Neurological Biomaterials \& Cancer Therapeutics, Wallace H Coulter Department of Biomedical Engineering, Georgia Institute of Technology/Emory University, 3108 UA Whitaker Bldg, 313 Ferst Drive, Atlanta, GA 30332-0535, USA = Tel. : +1 4043855038 m Fax: +1 4043855044

nravi@bme.gatech.edu

One of the holy grails of cancer therapy has been to find unique 'markers' in tumors that first, uniquely characterize the tumors in each individual patient, and second, are useful for diagnosis or therapy. Here, we suggest that while biomarkers are very useful in characterizing individual tumor cells, there is utility in describing tumors in a manner that captures their microenvironment, and such a description might be termed a 'physiomarker' as opposed to the more reductionist 'biomarker'. We further suggest that the angiogenic state of tumors, as represented by the leakiness of tumor blood vessels, might be a useful physiomarker, in that it integrates many underlying tumor characteristics such as VEGF and VEGF receptor (VEGFR) 2 and HIF-1 $\alpha$ expression - all factors shown to be useful in defining cancer individually. We argue that the physiomarker of tumor vessel leakiness is useful not only to characterize tumors and their aggressiveness, but also to determine the dosing of systemically administered drugs to these tumors.

When one explores options to personalize therapy for breast cancer specifically, it is useful to consider the major therapeutic approaches available today. Surgical resection, chemotherapy and radiation therapy form the cornerstones of all cancer therapy today. For breast cancer, the National Cancer Institute summarizes treatment options based on tumor staging, and these include surgery (ranging from breast-conserving to radical mastectomy) and adjuvant regimes of chemotherapy and radiation. However, as noted by Recht et al. [1], the optimal sequence for the three major interventional approaches is not yet clear. As many as 36\% of patients in a randomized trial who received chemotherapy first showed complete clinical response. Among the patients who did not show complete clinical response, practically all patients could be treated with a less radical surgical procedure. This has led to the notion of neoadjuvant chemotherapy emerging as common practice for treatment of locally advanced breast tumors, specifically primary tumors larger than $5 \mathrm{~cm}$ - stage T3 [2]. Interestingly, in addition to treating large tumors, neoadjuvant therapy is increasingly being used for smaller, operable tumors [3]. The National Surgical Adjuvant Breast and Bowel Project (NSABP) Protocol B-18 compared neoadjuvant to adjuvant chemotherapy in patients with operable, palpable breast cancer (T1-3; N0-1; M0) [4]. Neoadjuvant therapy followed by resection yielded patient survival equivalent to resection followed by adjuvant therapy. However, the neoadjuvant therapy facilitated $12 \%$ more lumpectomies (in lieu of mastectomies) [4].

Two important conclusions can be drawn from this study. First, there appears to be no additional risk in administering neoadjuvant therapy for operable tumors. In the USA, there are 147 active clinical trials evaluating neoadjuvant chemotherapeutic protocols for primary breast cancer, and of these, 26 trials are evaluating neoadjuvant therapy for operable tumors [101]. Second, in the NSABP trial comparing neoadjuvant and adjuvant therapy, there were $12 \%$ less mastectomies when neoadjuvant therapy was used, resulting in organ preservation and all the attendant benefits [2,4]. Given these advantages, the question arises - what is limiting the use of neoadjuvant therapy relative to adjuvant chemotherapy, and where is the need for 'personalization'? 
Breast cancer patients represent a very heterogeneous population with several parameters (including tumor stage, tumor vasculature status, hormonal status, aggressiveness of tumor, tumor type and resistance to administered drug) affecting the outcome of therapies [5]. Thus, in spite of the wide range of chemotherapy options available, it is effective only in a subset of cancer patients [6]. Given that neoadjuvant chemotherapy typically involves six cycles of therapy lasting approximately 6 months, it would be extraordinarily useful if one were able to predict the patient responsiveness to systemically administered chemotherapeutics so that potentially nonresponsive patients can undergo surgery, radiation or other therapeutic options.

We suggest that one critical variable affecting patient-to-patient variation in chemotherapy response can be traced to differences in drug dosing to tumors due to differences in the angiogenic status of each individual tumor. This is because the dosing of systemically delivered drugs to tumors is critically dependent on the access that these agents have to tumors via the so-called leaky vasculature of the tumor complex microvasculature network [7]. However, it is well-known that the extent of tumor vasculature leakiness differs among same-type tumors as a function of tumor stage, and there is patient-topatient variability even when patients are in the same tumor 'stage' $[8,9]$. One could hypothesize, therefore, that tumor vascular permeability status is a critical determinant of the uptake of systemically administered chemotherapeutics.

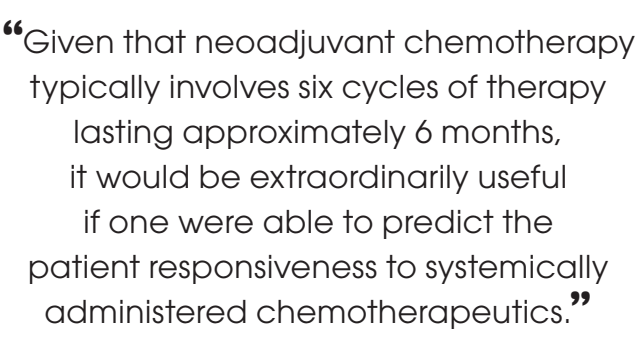

The advantage of defining these physiomarkers for tumors is that they lend themselves to determination noninvasively using image-based approaches. Currently, dynamic contrast-enhanced MRI using gadolinium chelates as contrast agents is employed in the clinic to characterize tumor blood vessels. However, unfortunately these free-molecule agents display a nonselective, promiscuous leak through the endothelium of most normal and pathological blood vessels, and therefore do not specifically characterize tumor vascular leakiness.
In a recent study, we demonstrated that $100 \mathrm{~nm}$ liposomal nanocarriers encapsulating iodinated contrast agents (termed as nanocontrast) generated sufficient contrast to quantify their extravasation through the leaky vasculature of a rat breast tumor model (MAT B III tumors) using mammography, and that there was significant variability in the tumor vascular permeability as determined by nanocontrast extravasation. Furthermore, when the same tumors were treated with Stealth liposomal doxorubicin (SLD), the efficacy correlated strongly with the extravasation of nanocontrast. Importantly, the extravasation of the nanocontrast agent, as quantified by standard mammography, predicted SLD efficacy by its ability to quantify tumor vessel 'leakiness' noninvasively. The nanocontrast probe, by virtue of its physical properties (size and surface charge) being very similar to those of SLD, was able to probe those physical properties of the tumor vasculature that controlled the localization of SLD, and thus inform the therapeutic protocol.

We therefore suggest that the concept of physiomarker, analogous to the now ubiquitous term biomarker, and the less often encountered chemomarker, is a useful, functional way to characterize tumors in a way that captures the aggregate, integrated consequences of several underlying biomarkers. Thus, while a biomarker has come to mean a biological molecule that can be associated with a particular condition, or disease state, and a chemomarker means a chemical property (such as $\mathrm{pH}$ ) that is associated with a particular condition, a physiomarker is a physical property (such as vascular leakiness) that can be associated with a particular condition. Furthermore, the physiomarker tumor vessel permeability is a significant determinant of drug dosing to tumors, and may have important clinical implications for a personalized diagnosis of the state of individual tumors, as well as for their treatment.

Financial \& competing interests disclosure

Some of the nanoscale contrast agents for CT imaging developed by the authors have been licensed by a startup company, Marval Biosciences Inc., in which the authors have a financial interest. The authors have no other relevant affiliations or financial involvement with any organization or entity with a financial interest in or financial conflict with the subject matter or materials discussed in the manuscript apart from those disclosed.

No writing assistance was utilized in the production of this manuscript. 


\section{Bibliography}

1. Recht A, Come SE, Henderson IC et al: The sequencing of chemotherapy and radiation therapy after conservative surgery for early-stage breast cancer. N. Engl. J. Med. 334(21), 1356-1361 (1996)

2. Buzdar AU: Preoperative chemotherapy treatment of breast cancer - a review. Cancer 110(11), 2394-2407 (2007).

3. Fisher B, Jeong JH, Dignam J et al: Findings from recent National Surgical Adjuvant Breast and Bowel Project adjuvant studies in stage I breast cancer. J. Natl Cancer Inst. Monogr. (30), 62-66 (2001).

4. Wolmark N, Wang J, Mamounas E, Bryant J, Fisher B: Preoperative chemotherapy in patients with operable breast cancer: nine-year results from National Surgical Adjuvant Breast and Bowel Project B-18. J. Natl Cancer Inst. Monogr. (30), 96-102 (2001).

5. Muller M, Mader RM, Steiner B et al.: 5-fluorouracil kinetics in the interstitial tumor space: clinical response in breast cancer patients. Cancer Res. 57(13), 2598-2601 (1997).
6. Harrington KJ, Mohammadtaghi S, Uster PS et al.: Effective targeting of solid tumors in patients with locally advanced cancers by radiolabeled pegylated liposomes. Clin. Cancer Res. 7(2), 243-254 (2001).

7. Jain RK: Transport of molecules, particles, and cells in solid tumors. Annu. Rev. Biomed. Eng. 1, 241-263(1999).

8. Fukumura D, Jain RK: Tumor microenvironment abnormalities: causes, consequences, and strategies to normalize. J. Cell. Biochem. 101(4), 937-949 (2007) .

9. Yuan F, Chen Y, Dellian M et al.: Time-dependent vascular regression and permeability changes in established human tumor xenografts induced by an anti-vascular endothelial growth factor/vascular permeability factor antibody. Proc. Natl Acad. Sci. USA 93(25), 14765-14770 (1996).

\section{Website}

101. US NIH: ClinicalTrials.gov website www.clinicaltrials.gov (Accessed 26 October 2008).

\section{Affiliations}

- Ananth Annapragada

School of Health Information Sciences,

University of Texas at Houston, Houston,

TX, USA

Tel.: +1 7135003982

Fax: +1 7135003929

Ananth.Annapragada@uth.tmc.edu

- Ravi V Bellamkonda

Neurological Biomaterials \& Cancer Therapeutics, Wallace H Coulter Department of Biomedical Engineering, Georgia Institute of Technology/Emory University, 3108 UA Whitaker Bldg, 313 Ferst Drive, Atlanta, GA 30332-0535, USA

Tel.: +1 4043855038

Fax: +1 4043855044

ravi@bme.gatech.edu 